\section{Acknowledgments}

This work was supported by the Juvenile Diabetes Research Foundation (JDRF), NIH, Leona M. and Harry B. Helmsley Charitable Trust, and Burroughs Wellcome Fund. We would also like to thank Kellsey Johannes and Imran Khan for helpful comments on a draft of this commentary.

Address correspondence to: Mark S. Anderson, UCSF Diabetes Center, 513 Parnassus Ave. HSW 1114, Box 0540, San Francisco, California 94143, USA. Phone: 415.502.8052; Fax: 415.564.5813; E-mail: manderson@diabetes.ucsf.edu.
1. Cooper LT. Myocarditis. N Engl J Med. 2009; 360(15):1526-1538

2. Rose NR. Myocarditis: infection vs. autoimmunity. J Clin Immunol. 2009;29(6):730-737.

3. Anderson MS, Bluestone JA. The NOD mouse: a model of immune dysregulation. Annu Rev Immunol. 2005;23:447-485.

4. Elliott JF, et al. Autoimmune cardiomyopathy and heart block develop spontaneously in HLA-DQ8 transgenic IA $\beta$ knockout NOD mice. Proc Nat Acad SciUS A. 2003;100(23):13447-13452.

5. Taylor JA, Havari E, McInerney MF, Bronson R, Wucherpfennig KW, Lipes MA. A spontaneous model for autoimmune myocarditis using the human MHC molecule HLA-DQ8. I Immunol. 2004;172(4):2651-2658.

6. Hayward SL, Bautista-Lopez N, Suzuki K, Atrazhev A, Dickie P, Elliott JF. CD4 T cells play major effector role and CD8 T cells initiating role in spon- taneous autoimmune myocarditis of HLA-DQ8 transgenic IAb knockout nonobese diabetic mice. J Immunol. 2006;176(12):7715-7725.

7. $\mathrm{Lv} \mathrm{H}$, et al. Impaired thymic tolerance to $\alpha$-myosin directs autoimmunity to the heart in mice and humans. J Clin Invest. 2011;121(4):1561-1573.

8. DeVoss JJ, et al. Effector mechanisms of the autoimmune syndrome in the murine model of autoimmune polyglandular syndrome type 1.J Immunol. 2008;181(6):4072-4079

9. Shum AK, et al. Identification of an autoantigen demonstrates a link between interstitial lung disease and a defect in central tolerance. Sci Transl Med. 2009;1(9):9ra20.

10. Bonasio R, Scimone ML, Schaerli P, Grabie N, Lichtman AH, von Andrian UH. Clonal deletion of thymocytes by circulating dendritic cells homing to the thymus. Nat Immunol. 2006; 7(10):1092-1100

\title{
Niche competition and cancer metastasis to bone
}

\author{
Laura G. Schuettpelz ${ }^{1}$ and Daniel C. Link ${ }^{2}$ \\ 1Division of Hematology/Oncology, Department of Pediatrics, and 2Division of Oncology, Department of Medicine, \\ Washington University School of Medicine, St. Louis, Missouri, USA.
}

\begin{abstract}
The molecular basis for the preferential metastases of certain cancers to bone is not well understood. In this issue of the JCI, Shiozawa et al. provide compelling evidence that prostate cancer cells preferentially home to the osteoblastic niche in the bone marrow, where they compete with normal HSCs for niche support. Because signals from the niche may regulate tumor quiescence and sensitivity to chemotherapy, these observations have important implications for the treatment of metastatic prostate cancer in bone.
\end{abstract}

Bone is a common site of metastases for certain tumors, including breast cancer and prostate cancer ( $\mathrm{PCa}$ ). Approximately $70 \%$ of patients with $\mathrm{PCa}$ have bone metastases at the time of death (1). The molecular basis for this preferential growth in the bone marrow and the biological effects of the rich microenvironment in the bone marrow on cancer cell growth and survival are not well understood. In this issue, Shiozawa et al. provide compelling evidence that PCa cells preferentially home to the osteoblastic niche in the bone marrow, where they compete with normal HSCs for niche support (2). Because signals from the niche may regulate the quiescence and survival of PCa cells (and possibly sensitivity to chemotherapy), these observations have important implications for the treatment of metastatic bone cancer.

The bone marrow microenvironment plays a critical role in the maintenance

Conflict of interest: The authors have declared that no conflict of interest exists.

Citation for this article: J Clin Invest. doi:10.1172/ JCI57229. of HSC quiescence and self-renewal. HSCs preferentially localize in the bone marrow, either to a perivascular location or near the endosteum $(3,4)$. Although the stem cell niche in the bone marrow is likely to be complex, with contributions from endothelial cells, advential reticular cells, nestinpositive stromal cells (5), and CXCL12abundant reticular (CAR) cells $(6,7)$, current evidence suggests that osteoblast lineage cells are a key component of the endosteal niche and are required to maintain normal HSC function. Expansion of osteoblast lineage cells by genetic or pharmacologic means results in concurrent expansion of HSCs (8). Conversely, ablation of osteoblasts using a suicide gene results in a loss of HSCs (9).

\section{CXCL12/CXCR4 axis}

Although the signals generated by the stem cell niche that regulate HSCs are not fully understood, a key player is CXCL12 (also known as stromal-derived factor-1), a chemokine constitutively expressed at high levels in the bone marrow by osteo- blasts, endothelial cells, and other bone marrow stromal cells. CXCL12, primarily through interaction with its major receptor, CXCR4, regulates HSC quiescence and homing to the bone marrow $(10,11)$. Disruption of CXCL12/CXCR4 signaling is a key step in cytokine-induced HSC mobilization from bone marrow to blood (12). The importance of the CXCL12/ CXCR4 axis is shown by the success of the CXCR4 inhibitor plerixafor (AMD3100; Genzyme) to rapidly mobilize HSCs in humans (13). Other agents produced by stromal cells in the endosteal niche that have been implicated in the regulation of HSCs include angiopoietin-1, thrombopoietin, and mediators of Notch and Wnt signaling (14-16).

Certain tumors, including PCa cells, appear to have coopted the CXCL12/ CXCR4 signaling pathway to preferentially home to the bone marrow. Whereas CXCR4 expression is low or absent in many normal tissues, it is expressed at high levels in more than 23 different cancers, including breast cancer, ovarian cancer, and PCa (17).

Importantly, inhibition of CXCR4 signaling has been shown to reduce metastatic disease of multiple tumor types in mouse xenograft models. The growth and metastasis of PCa cells injected into nude mice, for example, was inhibited by a neutralizing antibody to CXCR4 (15). Similarly, treatment of mice with small-molecule 


\section{A Baseline}

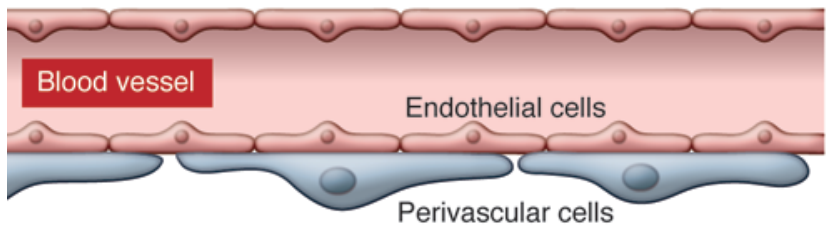

Perivascular cells

\section{Osteoblast niche}

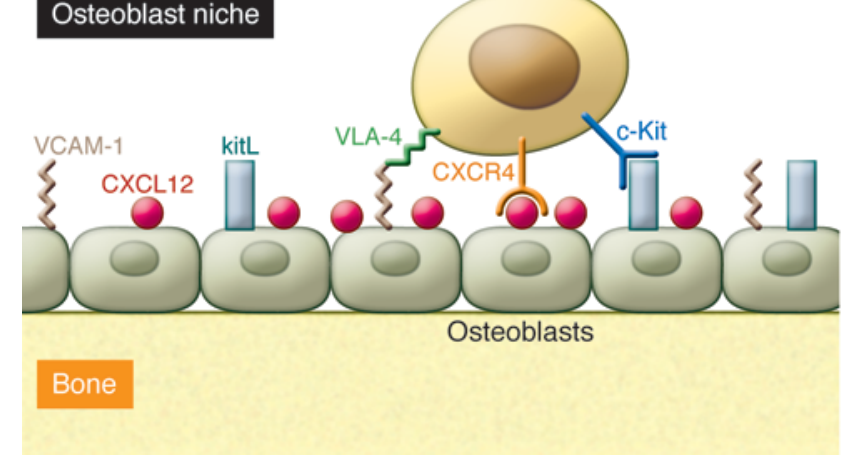

\section{B Prostate cancer}
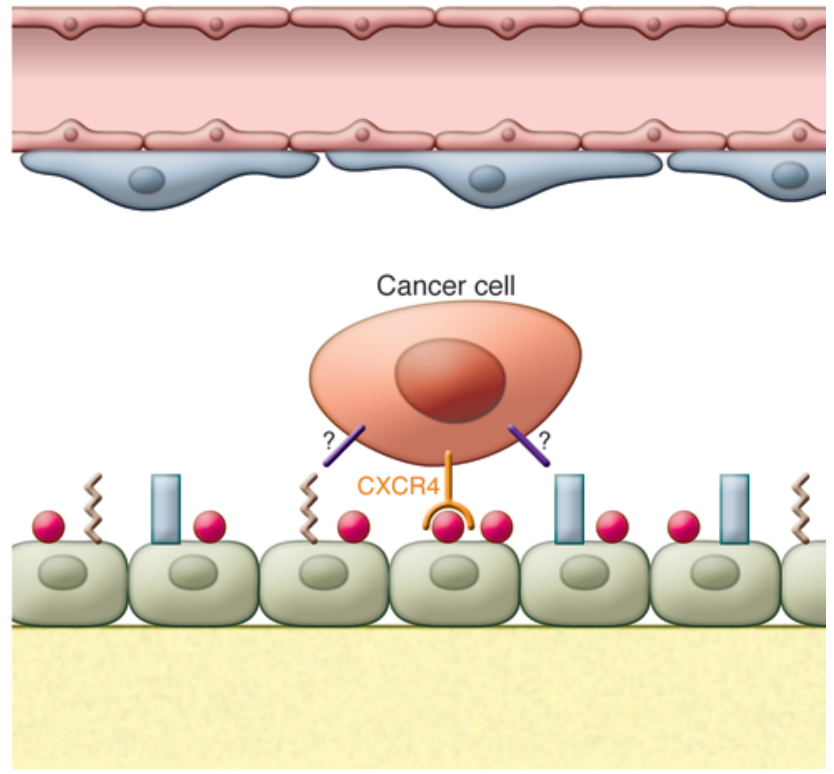

Figure 1

Tumor cells compete for the HSC niche. (A) HSCs reside in perivascular and endosteal niches within the bone marrow. Osteoblast lineage cells produce factors, including CXCL12, VCAM-1, and c-Kit ligand (kitL; also known as stem cell factor), that retain HSCs in the marrow and help maintain their quiescence and self-renewal capacities. (B) PCa cells, by coopting the CXCL12/CXCR4 axis, compete with normal HSCs for residence in the niche. Signals from the niche that promote the quiescence and self-renewal of HSCs may likewise maintain tumor cells in a more stem-like state, facilitating marrow metastasis and resistance to chemotherapy.

inhibitors of CXCR4 reduced the metastatic burden of transplanted breast cancer cells $(18,19)$.

\section{Sharing a niche}

Although tumor cells and HSCs use similar homing mechanisms, it has not been clear whether tumor cells directly occupy the HSC niche. Shiozawa et al. now provide compelling evidence that prostate tumor cells directly compete with HSCs for residence in the endosteal niche (2). Using a PCa xenograft model, they showed reduced HSC number and function in mice with micrometastases. Moreover, coinjection of PCa cells (but not control nonmetastatic transformed prostate epithelial cells) with normal murine or human HSCs decreased their engraftment in the bone marrow. Importantly, multiphoton imaging demonstrated that transplanted HSCs and PCa cells localized to the same (endosteal) region of the bone marrow. Perhaps most convincingly, they show that manipulation of the osteoblast niche affected the development of metastases: treatment with parathyroid hormone, which expands osteoblasts, increased the metastatic burden, whereas ablation of osteoblasts was associated with a reduced number of metastases.
There are several important clinical implications of these findings. First, displacement of normal HSCs from the niche by cancer cells may contribute to the peripheral cytopenias (e.g., neutropenia and anemia) that are common in patients with metastatic cancer. Second, because signals from the endosteal niche contribute to HSC quiescence and survival, it is possible that cancer cells located in the niche may acquire a more quiescent and stem-like phenotype, perhaps facilitating dormancy. Consistent with this possibility, disseminated tumor cells are commonly found in the bone marrow of men with PCa at the time of prostatectomy, and persistence of these cells after surgery is associated with an increased risk of recurrence (20). Third, the study by Shiozawa et al. predicts that agents that mobilize HSCs from the niche may also mobilize cancer cells; indeed, treatment of mice with the small-molecule CXCR4 antagonist AMD3100 or G-CSF, both potent mobilizing agents for HSCs, led to egress of PCa cells from the bone marrow (2).

\section{Evolving strategies}

Perhaps the most exciting aspect of this work is that it suggests potential strategies to disrupt cancer/stromal cell interactions in the bone marrow to sensitize cancer cells to chemotherapy (Figure 1). Although direct experimental evidence is lacking, signals from the osteoblast niche are predicted to induce cancer cell quiescence and provide survival signals, rendering cells resistant to chemotherapy. It follows that mobilization of cancer cells out of the niche using AMD3100, G-CSF, or other mobilizing agents may render the cells more sensitive to chemotherapy. In fact, there is evidence that treatment with AMD3100 sensitizes acute myeloid leukemia cells to chemotherapy (21), and phase I/II clinical trials of CXCR4 inhibitors (CTCE9908, British Canadian BioSciences Corp.; MSX-122,Metastatix Inc.) have been initiated for patients with refractory metastatic solid tumors. Caution is advised in using CXCR4 antagonists, however, as long-term disruption of the HSC niche could have clinically relevant effects on hematopoiesis and could potentially increase metastases to other anatomic sites.

Several important questions are raised by this study. Experiments were performed in immunodeficient mice using a PCa cell line. Do primary PCa cells also engraft the osteoblast niche in human bone marrow? Do other cancers with a predilection for metastases to bone also home to the osteo- 
blast niche? Finally, studies are needed to better characterize the biological effects of the osteoblast niche on the survival, quiescence, and sensitivity to chemotherapy of cancer cells. Ultimately, identification of the niche signals that regulate cancer cell phenotype may provide targeted strategies to render metastatic bone cancers more susceptible to chemotherapy.

Address correspondence to: Daniel C. Link, Division of Oncology, Department of Medicine, 660 S. Euclid Avenue, Campus Box 8007, St. Louis, Missouri 63110, USA. Phone: 314.362.8771; Fax: 314.362.9333; E-mail:dlink@dom.wustl.edu.

1. Roodman GD. Mechanisms of bone metastasis. NEngl J Med. 2004;350(16):1655-1664.

2. Shiozawa Y, et al. Human prostate cancer metastases target the hematopoietic stem cell niche to establish footholds in mouse bone marrow. J Clin Invest. 2011;121(4):1298-1312.

3. Kiel MJ, Yilmaz OH, Iwashita T, Terhorst C, Morrison SJ. SLAM family receptors distinguish hematopoietic stem and progenitor cells and reveal endothelial niches for stem cells. Cell. 2005;121(7):1109-1121.
4. Nilsson SK, Johnston HM, Coverdale JA. Spatial localization of transplanted hemopoietic stem cells: inferences for the localization of stem cell niches. Blood. 2001;97(8):2293-2299.

5. Mendez-Ferrer S, et al. Mesenchymal and haematopoietic stem cells form a unique bone marrow niche. Nature. 2010;466(7308):829-834.

6. Sugiyama T, Kohara H, Noda M, Nagasawa T. Maintenance of the hematopoietic stem cell pool by CXCL12-CXCR4 chemokine signaling in bone marrow stromal cell niches. Immunity. 2006; 25(6):977-988.

7. Omatsu Y, et al. The essential functions of adipoosteogenic progenitors as the hematopoietic stem and progenitor cell niche. Immunity. 2010;33(3):387-399.

8. Calvi LM, et al. Osteoblastic cells regulate the haematopoietic stem cell niche. Nature. 2003; 425(6960):841-846

9. Visnjic D, Kalajzic Z, Rowe DW, Katavic V, Lorenzo J, Aguila HL. Hematopoiesis is severely altered in mice with an induced osteoblast deficiency. Blood. 2004; 103(9):3258-3264.

10. Cashman J, Clark-Lewis I, Eaves A, Eaves C. Stromal-derived factor 1 inhibits the cycling of very primitive human hematopoietic cells in vitro and in NOD/SCID mice. Blood. 2002;99(3):792-799.

11. Peled A, et al. Dependence of human stem cell engraftment and repopulation of NOD/SCID mice on CXCR4. Science. 1999;283(5403):845-848.

12. Christopher MJ, Liu F, Hilton MJ, Long F, Link DC. Suppression of CXCL12 production by bone marrow osteoblasts is a common and critical pathway for cytokine-induced mobilization. Blood.
2009;114(7):1331-1339.

13. Liles WC, et al. Mobilization of hematopoietic progenitor cells in healthy volunteers by AMD3100, a CXCR4 antagonist. Blood. 2003; 102(8):2728-2730

14. Arai F, et al. Tie2/angiopoietin-1 signaling regulates hematopoietic stem cell quiescence in the bone marrow niche. Cell. 2004;118(2):149-161.

15. Yoshihara H, et al. Thrombopoietin/MPL signaling regulates hematopoietic stem cell quiescence and interaction with the osteoblastic niche. Cell Stem Cell. 2007;1(6):685-697.

16. Duncan AW, et al. Integration of Notch and Wnt signaling in hematopoietic stem cell maintenance. Nat Immunol. 2005;6(3):314-322.

17. Balkwill F. The significance of cancer cell expression of the chemokine receptor CXCR4. Semin Cancer Biol. 2004;14(3):171-179.

18. Liang $Z$, et al. Inhibition of breast cancer metastasis by selective synthetic polypeptide against CXCR4. Cancer Res. 2004;64(12):4302-4308.

19. Huang EH, et al. A CXCR4 antagonist CTCE-9908 inhibits primary tumor growth and metastasis of breast cancer. J Surg Res. 2009;155(2):231-236.

20. Morgan TM, et al. Disseminated tumor cells in prostate cancer patients after radical prostatectomy and without evidence of disease predicts biochemical recurrence. Clin Cancer Res. 2009;15(2):677-683.

21. Nervi $B$, et al. Chemosensitization of acute myeloid leukemia (AML) following mobilization by the CXCR4 antagonist AMD3100. Blood. 2009;113(24):6206-6214.

\title{
Finding a needle in a haystack: whole genome sequencing and mutation discovery in murine models
}

\author{
Raajit K. Rampal and Ross L. Levine \\ Human Oncology and Pathogenesis Program and Leukemia Service, Department of Medicine, \\ Memorial Sloan-Kettering Cancer Center, New York, New York, USA.
}

\begin{abstract}
Acute promyelocytic leukemia (APL) is a malignancy of the bone marrow, in which there is a deficiency of myeloid cells and an excess of immature cells called promyelocytes. APL is most commonly caused by a translocation (15:17) and expression of the promyelocytic leukemia and the retinoic receptor $\alpha$ (PML-RARA) fusion product; however, the events that cooperate with PML-RARA in APL pathogenesis are not well understood. In this issue of the JCI, Wartman and colleagues use an innovative approach to find other relevant mutations in APL. They performed whole genome sequencing and copy number analysis of a well-characterized APL mouse model to uncover somatic mutations in Jak1 and lysine (K)-specific demethylase 6A (Kdm6a, also known as $U t x)$ in mice with APL and validated the ability of Jak1 mutations to cooperate with PML-RARA in APL. The findings implicate the JAK/STAT pathway in the pathogenesis of APL and illustrate the power of whole genome sequencing to identify novel disease alleles in murine models of disease.
\end{abstract}

Conflict of interest: The authors have declared that no conflict of interest exists.

Citation for this article: J Clin Invest. doi:10.1172/ JCI57200.
Acute promyelocytic leukemia (APL) is a clinically and molecularly distinct subtype of acute myeloid leukemia that is distinguished by a recurrent chromosom- al translocation fusing chromosomes 15 and 17 . The $t(15: 17)$ translocation results in the fusion of the promyelocytic leukemia $(P M L)$ gene and the retinoic receptor $\alpha(R A R A)$ gene (PML-RARA). The PMLRARA fusion protein is thought to contribute to APL pathogenesis by dimerizing and binding DNA and repressing the transcription of RARA target genes through recruitment of corepressors. More recent work indicates that PMLRARA also is able to bind to alternate DNA sites and to interact with chromatin remodeling complexes involved in stem cell maintenance and that the PMLRARA protein undergoes posttranslational modifications (sumoylation and phosphorylation) that are required for APL initiation $(1,2)$. A detailed understanding of the role of the PML-RARA 